\title{
An efficient data reduction method and its application to cluster analysis
}

\author{
Jianpei Wang, ShihongYue*, Xiao Yu, Yaru Wang
}

School of Electrical Engineering and Automation, Tianjin University 300072, China

\begin{abstract}
Data reduction plays a very important role in the data mining field, but the existing methods haven't been able to efficiently identify all major features which are hidden in the large datasets. On some occasions, they even cause the loss of the original key features. In this paper, a new efficient measure was developed to reduce a given dataset and to uncover the major features by multiplying the defined absolute density with the defined local density of any data. These two kinds of densities were estimated with a fast grid-based bisecting method. To test its performance on feature reduction and sample reduction, a group of feature-different synthetic datasets and 24 benchmark datasets were used as examples and the clustering accuracy, runtime and separability among clusters were used as measurements. The results strongly proved the proposed method could fast reduce a dataset and identify the most important key features. Additionally, it also can effectively determine the optimal number of clusters by suppressing the noisy data and enhancing the separation among clusters.
\end{abstract}

Keywords : Data reduction; Dimensionality reduction; Sample Reduction; Clustering validity.

\section{Introduction}

New information technologies and data mining techniques have led to an explosion in assessable data, resulting in two fundamental problems: high dimensionality and massive data [1-3]. High dimensionality often means the prevalence of a large number of useless/ineffective features that tend to keep key features hidden, while redundant data not only take up storage and memory space, but also affect classification accuracy and decision correctness. Data reduction under these conditions becomes a necessary technique for discovering key properties wherein irrelevant or unimportant information is reduced from a massive dataset. The main operating principles and methods behind data reduction are dimensionality reduction and sample reduction [4-6].

In the past decades, data reduction techniques have been mainly focused on the feature reduction that can be categorized as linear and nonlinear groups. The most widely used feature reduction method is the Principal Component Analysis (PCA) [7]. It projects the data into a lower-dimensional space where the samples can be separated by a linear classifier (under the condition of that the samples can be separated by density in the input space). Thus the usage of PCA is based on the assumption of the linear separability of the input data. In the case of datasets with nonlinear separability, an efficient method is the Locally Linear Embedding (LLE) [8]. LLE has recently been proposed as a general method for dimensionality reduction of high-dimensional nonlinear datasets. Besides these, more recently, some novel dimensionality reduction algorithms have been proposed for image clustering and multi-view learning [9-11]. Based on the patch alignment framework, a novel semi-supervised dimensionality reduction was introduced for multi-view data analysis [9]. The hypergraph was used to integrate different relationships from different views to overcome the over-simplified assumption of pairwise relationships among data in most methods. After that, a sparse patch alignment framework [10] was proposed for the embedding of data underlying in multiple manifolds, which adopt an optimization strategy for constructing local patches instead of a fixed neighborhood size. For the semi-supervised multi-task feature selection problems, a manifold regularized multi-view feature selection method was presented in [11]. It exploited the label information, label relationship, data distribution, as well as correlation among different kinds of features simultaneously. More review can be found in [12-14].

1 
However, as the amount of information increases exponentially, reducing the total amount of massive data becomes necessary and inevitable. Sample reduction provides an efficient solution to this problem. The goal of sample reduction is to separate the unimportant details from the essential structures hidden in the data, while the structure of the data can be shown by various clusters. Thus, the clusters at various levels are a mostly visual approach, and are intended to maximize insight into a dataset, as well as uncover the structure of the data. In general, any useful algorithm is based on a set of requirements that are intended to advance the use of the data reduction as a data structure discovery technique; the technique must also be practical in terms of computational effort with the following characteristics:

(i) It always is validated for arbitrary data structures such as density-different, size-different, and irregular clusters after data reduction.

(ii) It must not be affected by partially overlapped clusters. Inversely, the reduced dataset should have better separation among clusters than the original dataset. Thus the overlapped parts among clusters must be reduced as data reduction proceeds.

(iii) It should be practically unsupervised and not utilize parameters defined for unrealistic prior information.

(iv) It should be non-iterative. An iterative algorithm usually is time-consuming against the linear time complexity. Therefore, a partitioning dataset is favorable as far as clustering efficiency is considered.

As such, there are primarily two classes of sample reduction methods: sampling and vector quantization [12-15]. Sampling is a method that is generally used to quickly reduce data in an investigated dataset; it is popular due to its simplicity and easy execution, as random sampling (RS) [16]. The disadvantage of this method is that it is not capable of recovering data structures and features, which can result in loss of key information in the data reduction process. Another class of data reduction method involves the use of learning vector quantization (LVQ) algorithms [17]. LVQ is different from sampling methods in that the learning process is intuitive and involves simple implementation. Originally, the class of methods is designed to overcome the limitation of RS methods by greatly adding the runtime of data reducing process. But the class of methods is not completely unsupervised and is limited in its ability to recover key features in the data reduction process [18].

To resolve the above problems, in this paper, we proposed a new data reduction method based on a fast grid-based bisecting (GB) method [19]. By reformulating the GB algorithm, a new measure was calculated by optimally multiplying the absolute density and local density of the data. Even though the absolute density in our method is similar to other density measures used in many other clustering algorithms, e.g., DBSCAN [20], but we proposed a new method that could estimate the absolute density much faster in an unsupervised way. Most importantly, our method doesn't need any user-specified threshold. Therefore it can be used to analyze any data. Then a local density is also proposed to overcome the problem that the existing absolute density cannot abstract the local clustering structure of the data. By multiplying the two densities, the proposed new data reduction method not only efficiently recovers the major feature, but also rapidly reduces the amount of data and greatly suppresses noisy data. Its non-iterative and grid-based data process has a linear time complexity. Thus it can greatly facilitate the computational loads of the data reducing process.

We further apply the proposed method to an important problem in cluster analysis, that is, quantitative validation of the used clustering results, including determining the optimal number of clusters and their configurations. In past decades, research has focused on various validity indexes. Some extensive and good overviews of clustering algorithms can be found in the literature [21-25]. Estimating the correct number of clusters by cluster validity indexes in cluster analysis is highly susceptible to data structure, separation among clusters and noisy data, so the correctness of the estimated number of clusters is difficult to be guaranteed. In this paper, the validity index is used to estimate the number of clusters in the reduced datasets. A group of synthetic datasets and 24 benchmark datasets are used to validate the proposed method. 


\section{Related work}

In this section, we introduced five related algorithms, including two clustering algorithms, $C$-MEANS and GB, and four data reduction algorithms, RS, LBG, PCA and LLE, as well as five typical cluster validity indexes as following.

\section{1. $C$ - MEANS and GB algorithms}

Assume that $X=\left\{x_{1}, x_{2}, \ldots, x_{n}\right\}$ is a set of $n$ data in a $d$-dimensional data vector space, and $C_{1}, C_{2}, \ldots$, $C_{c}$ are $c$ sets, then the belongingness of the $j$-th data vector in $X$ to the $i$-th set can be represented by a binary membership function as

$u_{i j}=\left\{\begin{array}{ll}1, & x_{j} \in C_{i} \\ 0, & x_{j} \notin C_{i}\end{array} \quad, \quad i=1,2, \ldots, c, \quad j=1,2, \ldots, n\right.$.

If any data vector in $X$ is only assigned to a unique set, then all vectors must be assigned into $c$ sets. This is called as a hard partitioning of $X$, satisfying

$X=C_{1} \cup C_{2} \cup \ldots \cup C_{c}, \quad C_{i} \cap C_{j}=\phi, i, j=1,2, \ldots, c$

Currently, the class of partitional clustering algorithms is designed based on objective function optimization, amongst which the $C$-MEANS algorithm [22] is well accepted and the most used clustering method because of its simplicity, robustness, and easy application. The detailed steps of the $C$-MEANS algorithm and its time complexity are shown in Table 1, where $c$ is the number of clusters, $t$ is the totally iterative times, and any cluster center (clustering prototype) is computed by

$v_{j}=\sum_{j=1}^{n} u_{i j} x_{j} \sum_{j=1}^{n} u_{i j}, i=1,2, \ldots, c$

Table 1 C-MEANS algorithm

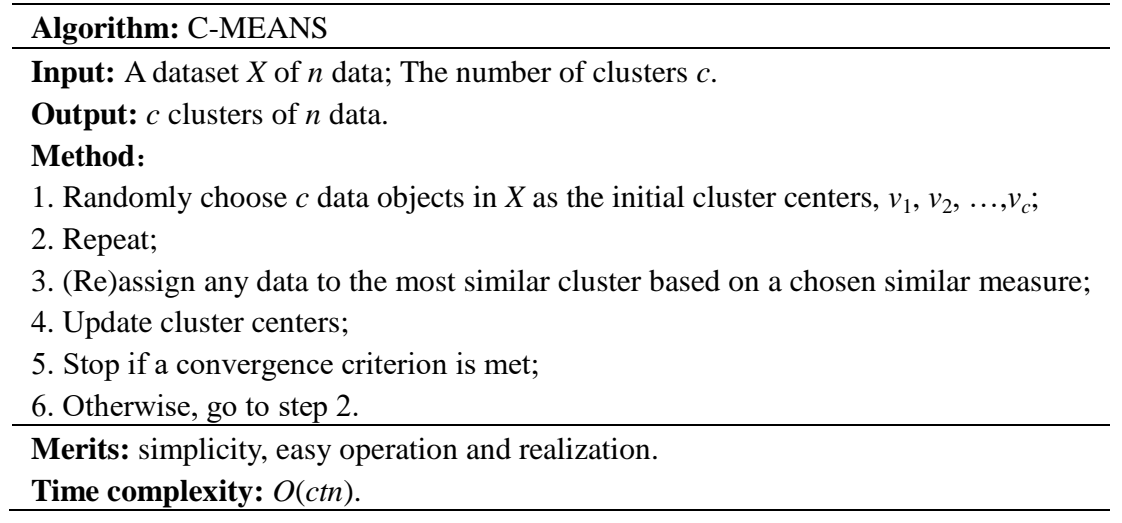

In [19] a fast grid-based bisecting (GB) algorithm has been applied to partition any dataset into a group of grids. GB algorithm successively bisects every edge of any grid in each dimension by turns; the dataset is then partitioned into $2,2^{2}, \ldots, 2^{J}$ grids after $J$ times, where $J$ is the maximal bisecting times when no grid contains two points. Fig. 1 shows the bisecting process by a simple example by a dataset of 26 data points.

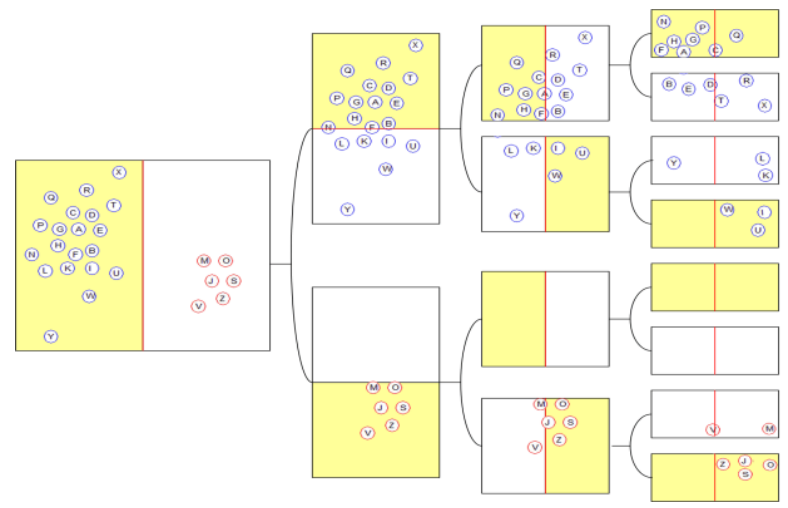

Fig.1. A bisecting process of a dataset and yellow sub-grids with more points. 
Table 2 shows the general bisecting process of GB. The time complexity of GB is linearly $O(n)$ while C-MEANS is $O(c t n)$. So GB is much faster than C-MEANS.

Table 2 The bisecting process in the GB algorithm

\begin{tabular}{l}
\hline Algorithm: GB \\
Input: A dataset $X$ containing $n$ data. \\
Output: A set of nonempty grids that contain all $n$ data. \\
Method: \\
1.Solve the minimal grid that encloses all data objects in $X$ and denoted as GRID; \\
2.Choose a dimension to bisect by turns; \\
3.Bisect any grid in the chosen dimension to two sub-grids; \\
4.Assign all data in $X$ to the set of grids in any bisecting process; \\
5.Stop bisecting if any grid contains only one data point; \\
6. Find all high-density grids for further clustering.
\end{tabular}

\subsection{Four typical data reduction algorithms}

Four typical data reduction algorithms, two for sample reduction and the other two for dimensionality reduction, are introduced below.

1) Two sample reduction methods. Random sampling (RS) is a method of selecting $n$ units out of all $N$ samples to make sure every distinct sample has an equal chance to be selected. In practice, a simple random sampling is processed unit by unit. RS is a simple and easily realized method with low time complexity. But RS cannot abstract the major data structures and features hidden in data. Alternatively, vector Quantization (VQ) is one example of competitive learning [14]. The goal of VQ is to discover structures in the data through data reduction. The results can be used for data encoding and compression. In 1980, a famous LBG algorithm [17] on VQ was proposed based on a training sequence, which bypasses the need for multidimensional integration. The effect of LBG was reported as similar to that of $C$-MEANS when both were applied to find the data structure of an investigated dataset [26]. Thus LBG can extract the major features better than the RS algorithm. The LBG algorithm is summarized below.

Table 3 The LBG algorithm

Algorithm: LBG
Input: A dataset $X$ containing $n$ data objects.
Output: A set of $K$ representatives that contain all $n$ data.
Method:
1. Fix the distortion threshold $\varepsilon$ as to be a small positive number.
2. Set the number of code vectors $K=1$.
Let the code vector be the mean of all samples and compute the total distortion.
3. Multiply any code vector by the disturbing factor $(1+\varepsilon)$ and $(1-\varepsilon)$ to split the
code vector into two new vectors.
4. Iteration (Initial distortion is the one in step 2):
1) Find the nearest code vector to any sample to assess the sample.
2) Compute the mean of all samples that belong to the same code vector as the
new code vector of this coding region.
3) Compute the current distortion.
if it is larger than $\varepsilon$, go to 1$)$, otherwise, it is the final distortion
and the corresponding code vectors are the final ones.
5. Repeat Steps 3 and 4 until the desired number of code vectors is obtained.

2) Two dimensionality reduction methods. Principal Component Analysis (PCA) [7] is the most widely used linear method for dimensionality reduction. It performs a linear mapping of a data to a lowerdimensional space to maximize its representationality. To do this, the covariance matrix of the data is constructed and the eigenvectors of it are computed. Then the eigenvectors corresponding to the largest eigenvalues (the principal components) are used to reconstruct a large fraction of the variance of the original 
data. Because the first few eigenvectors can often be interpreted as the large-scale physical behavior of the system, the original space can be reduced to the new lower dimensional space spanned by these few selected eigenvectors. Table 4 shows the steps of the PCA algorithm.

On the other hand, Locally Linear Embedding (LLE) [8] is a classical nonlinear technique for dimensionality reduction. Given a dataset $X$, LLE begins by finding $K$ nearest neighbors of certain sample $x_{i}$ in $X$. It then computes a set of weights for each point that can best describe the point as a linear combination of its neighbors. The weights $W_{i j}$ are optimized by minimizing the following reconstruction error,

$\varepsilon(W)=\sum_{i}\left|x_{i}-\sum_{j} W_{i j} x_{j}\right|^{2} \quad$ s.t. $\sum_{j} W_{i j}=1$

where $W_{i j}=1$ when $x_{j}$ belongs to the neighborhood of $x_{i}$, otherwise, $W_{i j}=0$. LLE uses an eigenvector-based optimization technique to find the low-dimensional embedding of points in the embedded space in which each point can be described as the same linear combination of its neighbors. Suppose the low-dimensional embedding is $Y$, the embedding $y_{i}$ can be optimized by minimizing the embedding cost function, $\Phi(Y)=\sum_{i}\left|y_{i}-\sum_{j} W_{i j} y_{j}\right|^{2}$

(5) can be minimized by seeking the smallest eigenmodes of the sparse symmetric matrix as $M_{i j}=\delta_{i j}-W_{i j}-W_{j i}+\sum_{k} W_{k i} W_{k j}$

where $\delta_{i j}$ is 1 if $i=j$ and 0 if otherwise. LLE tends to handle non-uniform sample densities poorly because there is no fixed unit to prevent the weights from drifting as various regions differ in sample densities. The LLE algorithm is also shown in Table 4.

Table 4. Illustration of PCA and LLE algorithms

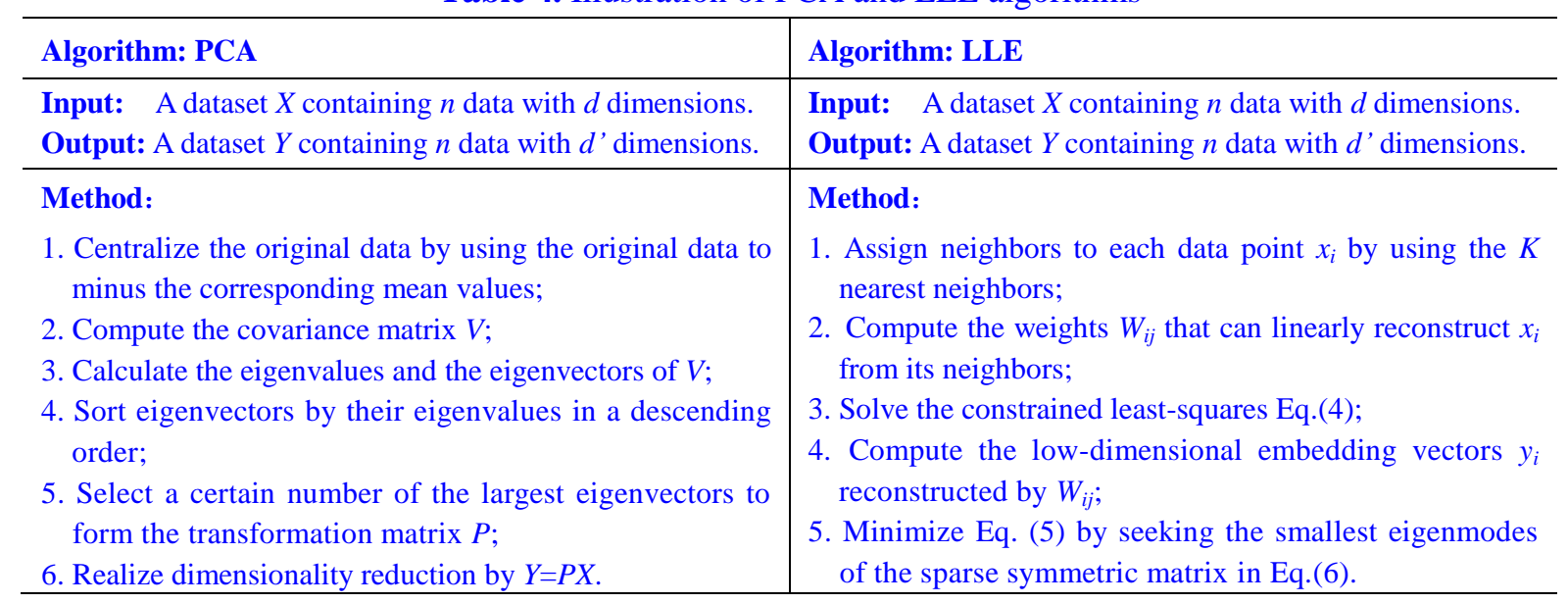

\subsection{Typical validity indexes}

Generally, the partitioning of a dataset is performed by a clustering algorithm, and the partitioning results are evaluated by the optimum of a function that uses the number of clusters as its variables. This function is called a validity index. Let $c$ be the number of clusters in the investigated dataset, and $v_{i}$ is the $i$-th cluster center, $i=1,2, \ldots, c$. In the following, each abbreviation is marked with an up or down arrow. The down arrow denotes that a lower value of that index means a "better" partition. The up arrow means exactly the opposite.

1) Davies-Bouldin index $(D B \downarrow)$ [27]. Let $\Delta_{i}$ be the $i$-th within-cluster distance measure; $\delta_{i j}$ is the betweencluster distance measure between $C_{i}$ and $C_{j}$. The simplified $D B$ index is defined as

$D B=\sum_{i=1}^{c} R_{i, q} / c$, s.t., $R_{i}=\max _{j, j \neq i}\left(\Delta_{i}+\Delta_{j}\right) / \delta_{i j}, \delta_{i j}=\left\|v_{i}-v_{j}\right\|, \Delta_{i}=\sum_{x \in C_{i}}\left\|x-v_{i}\right\| /\left|C_{i}\right|$

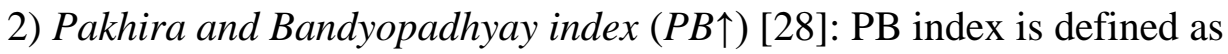

$$
V_{P B M F}=\left(\frac{1}{c} \times \frac{E_{1}}{J} \times D_{c}\right)^{2} \text {, s.t., } E_{1}=\sum_{j=1}^{n}\left\|x_{j}-v\right\|, D_{c}=\sum_{i, j=1}^{c}\left\|v_{j}-v_{i}\right\|, J=\sum_{i=1}^{c} \sum_{j=1}^{c}\left\|x_{j}-v_{i}\right\|
$$


where $v$ is the centroid of the dataset.

3) Calinski-Harabasz index $(\mathrm{CH} \uparrow)$ [29]: This $\mathrm{CH}$ index is a ratio-type index as

$C H(c)=\left(\frac{n-c}{c-1}\right) \frac{\sum_{C_{c} \in C}\left|C_{c}\right| d\left(\bar{C}_{c}, \bar{X}\right)}{\sum_{C_{c} \in C} \sum_{x_{i} \in C_{c}} d\left(x_{i}, \bar{C}_{c}\right)}$, s.t., $\bar{C}_{c}=\sum_{x_{i} \in C_{c}} x_{i} /\left|C_{c}\right|$, and $\bar{X}=\sum_{x_{i} \in X} x_{i} / n$

4) Silhouette index (Sil $\uparrow$ ) [30]: This index is a normalized summation-type index. The cohesion is measured based on the distance between all points in the same cluster and the separation is based on the nearest neighbor distance. It is defined as

$\operatorname{Sil}(c)=\sum_{C_{c} \in C} \sum_{x_{i} \in C_{c}} \frac{b\left(x_{i}, C_{c}\right)-a\left(x_{i}, C_{c}\right)}{n \max \left\{a\left(x_{i}, C_{c}\right), b\left(x_{i}, C_{c}\right)\right\}} \quad$ s.t., $a\left(x_{i}, C_{c}\right)=\sum_{x_{j} \in C_{c}} d\left(x_{i}, x_{j}\right) /\left|C_{c}\right|$, and $b\left(x_{i}, C_{c}\right)=\min _{C_{i} \in C \backslash C_{c}} \sum_{x_{j} \in C_{l}} d\left(x_{i}, x_{j}\right) /\left|C_{l}\right|$

5) Krzanowski-Lai index $(K L \uparrow)$ [31]: This index is based on the measurement of the within-cluster difference matrix of all samples. Let $\operatorname{tr} W(c)$ be the trace of the within-cluster difference matrix, and then $K L$ index can be defined as

$K L(c)=|\operatorname{Diff}(c)| /|\operatorname{Diff}(c+1)|, \quad$ s.t., $\operatorname{Diff}(c)=(c-1)^{2 / p} \operatorname{tr} W(c+1)-c^{2 / p} \operatorname{tr} W(c)$

where $p$ is the number of dimensions(variables).

Using the existing validity indexes is problematic because the following factors can greatly affect their correctness when a clustering algorithm is used:

1) Various clusters. Estimating the correct number of clusters by a cluster validity index in cluster analysis is highly susceptible to data structure (e.g., different densities/sizes and overlapped clusters), separation among clusters and noisy data. So the correctness of the estimated number of clusters is difficult to be guaranteed.

2) Noisy data. Noisy data may affect the clustering results for any clustering algorithm. If the rate of noisy data is higher than about $30 \%$ of total number of data, any clustering results is difficultly guaranteed [16].

Consequently, enhancing separation and suppressing noises by data reduction are not only can find major features in a dataset but also helpful to effectively determine the optimal number of clusters.

\section{New measure based on density and separation values}

In this section the absolute density and local density of any data is first defined, and thereby a new measure is computed. The new measure leads to a new data reduction method to reduce a dataset and improve the effectiveness of any validity index.

\subsection{Absolute and local densities}

Let $X=\left\{x_{1}, x_{2}, \ldots, x_{n}\right\}$ be an investigated dataset, $x_{j}=\left(x_{j 1}, x_{j 2}, \ldots, x_{j d}\right)^{T} \in R^{d}, G R I D$ be the minimal grid that encloses $X$. Assume $X$ is bisected by the GB algorithm, and after $T$ times of bisecting all data in $X$ are grouped into $n$ single-data grids, respectively. Thus there are totally $2^{T}$ grids and most of them are empty.

Definition 1. Absolute Density. Let $x_{j} \in X$. The absolute density of $x_{j}$ is defined as the minimal bisecting times of a grid that contains only $x_{j}$ itself, denoted as $\varepsilon_{j}$.

Clearly, more data the neighborhood of the data contains, larger absolute density the data has. Most clustering algorithms identify all clusters by their measures that are similar with the absolute density, such as DBSCAN, STING, and etc [25]. But our proposed density has the following three peculiar characteristics:

1) No parameter. The computation of defined density is perfectly unsupervised and without any prior parameter. As contrast, a threshold of any existing method has to be determined in advance. Different thresholds surely affect their density results.

2) Low runtime. The grid-based computation needs much less runtime than the existing algorithms in which the distances among points have to be computed repeatedly.

3) Discrete value. The absolute density of any data in $X$ must be one of $T$ discrete values, $1,2,3, \ldots, T$, when 6 
all data are partitioned into $n$ single-point grids after $T$ times of bisecting.

The absolute density cannot efficiently find all clusters, essentially such as density-different clusters, partially overlapped clusters, and etc. To reformulate the absolute density, we assume that a grid GRID $(k-1)$ that contains $x_{j}$ is bisected into a pair of sub-grids $\operatorname{GRID}(k, 1)$ and $\operatorname{GRID}(k, 2)$ at $k$-th time of bisecting, satisfying $|G R I D(k, 1)| \geqslant|G R I D(k, 2)|$, a binary function of $x_{j}$ is define as

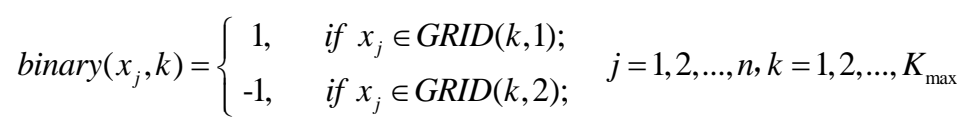

Definition 2. Local density. Let $x_{j} \in X$. The local density of $x_{j}$ is defined as

$\delta\left(x_{j}\right)=\min \left\{\sum_{k=1}^{\varepsilon_{j}} \operatorname{binary}\left(x_{j}, k\right), 1\right\}=\min \left\{\left(C_{\varepsilon_{j}}^{q_{j}}-C_{\varepsilon_{j}}^{\varepsilon_{j}-q_{j}}\right), 1\right\}, j=1,2, \ldots, n$

where $q_{j}$ is the number of " 1 " among $\varepsilon_{i}$ times of bisecting; the number of " 1 "is taken as the low bound of (13), and aims to avoid its value being a negative number. The sign " $C_{N}^{M}$ " is a combination number is computed as

$C_{N}^{M}=N(N-1) \ldots(N-M+1) / M(M-1) \ldots 3 \times 2 \times 1$.

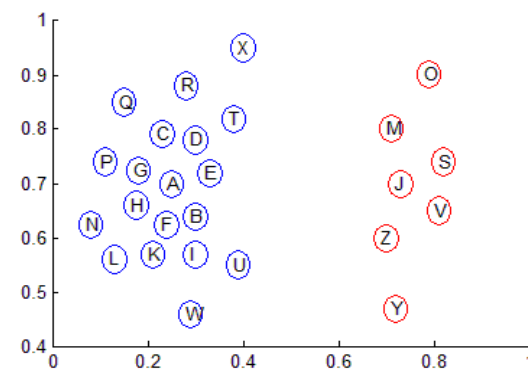

(a) Dataset of 26 points

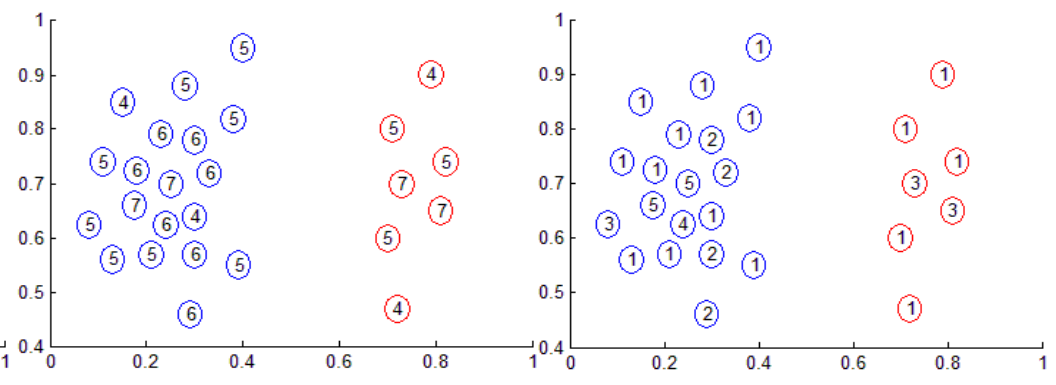

(b) Absolute densities of 26 points

(c) Local densities of 26 points

Fig.2. Effect of absolute and local densities of various points.

We find that the local maximums of absolute density are at points $\mathrm{A}, \mathrm{H}, \mathrm{J}, \mathrm{V}$, which are identifies as cluster centers. Those points C, D, G, E, and W still have high absolute density. But their local densities are very different from these centric points. For example, points $\mathrm{G}$ and A have almost the same absolute density but very different local densities. In fact, point $G$ is located in the transaction area from cluster center to boundary, many pairs of " 1 " and "-1"in Eq.(13) are summed up to zero. Specially, few points have large local density. The local densities of cluster center are anomalously larger than that of other points nearby.

\subsection{A new measure to evaluate the importance of any point}

An optimized measure preferably finds all clusters and greatly reduces the noisy data, as explained below.

Definition 3. Importance degree. Let $x_{j} \in X$. The importance degree $r_{j}$ is defined as

$r_{j}=\varepsilon_{j} \delta_{j}, j=1,2, \ldots, n$

Fig.3 (a) shows the plot of $\varepsilon_{i}{ }^{-} \delta_{j}$ in the dataset in Fig.2, as well as the plot of $r_{j}$ sorted in their decreasing orders in Fig.3(b).

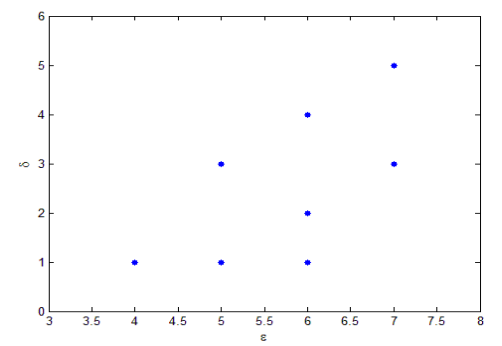

(a) $\varepsilon_{i}-\delta_{j}$ plot

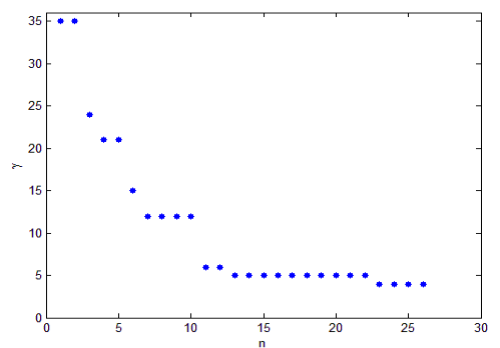

(b) $r_{j}$ plot

Fig.3. Effects of $\varepsilon_{i}-\delta_{j}$ and $r_{j}$. 
Fig. 3 shows that all points in a dataset can nearly be partitioned into three classes:

1) Core points: the 26 points just have 4 discrete values of absolute densities. However, the $\varepsilon_{i}-\delta_{j}$ plot cannot provide an order to rank all points. Inversely, the $r_{j}$ plot can utilize the complementarities of absolute density and local density to rank all points by their importance degrees.

2) Inner points: These points take up the majority of all points. They follow these core points and thus have high absolute but low local density. Thus their importance degrees are much lower than those core points. If these points are removed, cluster structures are hardly affected since these prototypes are kept in core points. 3) Boundary and noisy points. The boundary points are unimportant to the cluster structure. Inversely, when these points are removed, the separation among clusters can become clearer. Essentially, these noisy data and outliers must be suppressed and removed. These points apparently have very low importance degrees.

So importance degree can show the contributions of all points to major clustering structures. Hereafter, the proposed importance degree of Eq.(15) is called ID. Increasingly ordering all data by their IDs, the top $\beta \%$ data of small importance degrees are removed at the reduction rate of $\beta \%$. The ID-based data reducing method is called IDDR. For a dataset of $n$ data, IDDR mainly consists of three parts:

A) Assigning all points to a set of grids by $T$ times of bisecting;

B) Computing importance degrees of all points by their absolute and local densities;

C) Reducing the dataset at a specified rate.

In the first part the maximal number of grids is $2^{T}$ at most to $T$ times of bisecting for $n$ points, it needs $n\left(2^{T}\right)$ inquiring operations. The time complexity is $O\left(\log \left(n\left(2^{T}\right)\right)\right.$. On the other hand, according to the computational complexity conversion theory, the Euclidian distance computation of a pair of objects consists of about 60 1000 basic computational operations for diverse dimensions, while the inquiry of a pair of points consists of 10 basic computational operations. Consequently, the runtime in IDDR is about 6 120 times more than that of LBG. In the second and third parts, the computational effort just remains inquiry and comparison. Consequently, the total time complexity of IDDR is afforded in A), which is lower than linear time complexity. The IDDR algorithm is illustrated in Table 5 as follows.

Table 5 IDDR algorithm

\begin{tabular}{l}
\hline Algorithm: IDDR \\
\hline Input: A dataset $X$ of $n$ data. \\
Output: Reduced dataset with a reducing rate. \\
Method: \\
1. Bisect $X$ to $2^{T}$ grids such that any grid is a single-point or empty grid. \\
2. Compute $\varepsilon_{i}$ by inquiring the bisecting times of $x_{k}$ when $x_{k}$ falls into a single-point grid. \\
3. Compute $\delta_{j}$ of $x_{k}$ by (15) \\
4. Compute $r_{k}$ of $x_{k}$ by (16). \\
5. Decreasingly order all points by their importance degrees. \\
6. Reduce data by their importance degree from small to large. \\
\hline Time complexity: $O\left(\log \left(n\left(2^{T}\right)\right)\right.$ \\
\hline
\end{tabular}

\section{Experiments}

The proposed IDDR algorithm is evaluated by four criterions: feature extraction, separation among clusters, reduction runtime, and correctness of validity indexes. The four criterions are measured by contrast on the original and reduced datasets for all data reduction methods, respectively. In the comparison, the four most used data reduction methods, RS, LBG, PCA and LLE are applied. Experiments are performed in 3 synthetic datasets with different characteristics and 24 benchmark datasets in UCI [32]. Fig.4 shows the experimental flowchart. 


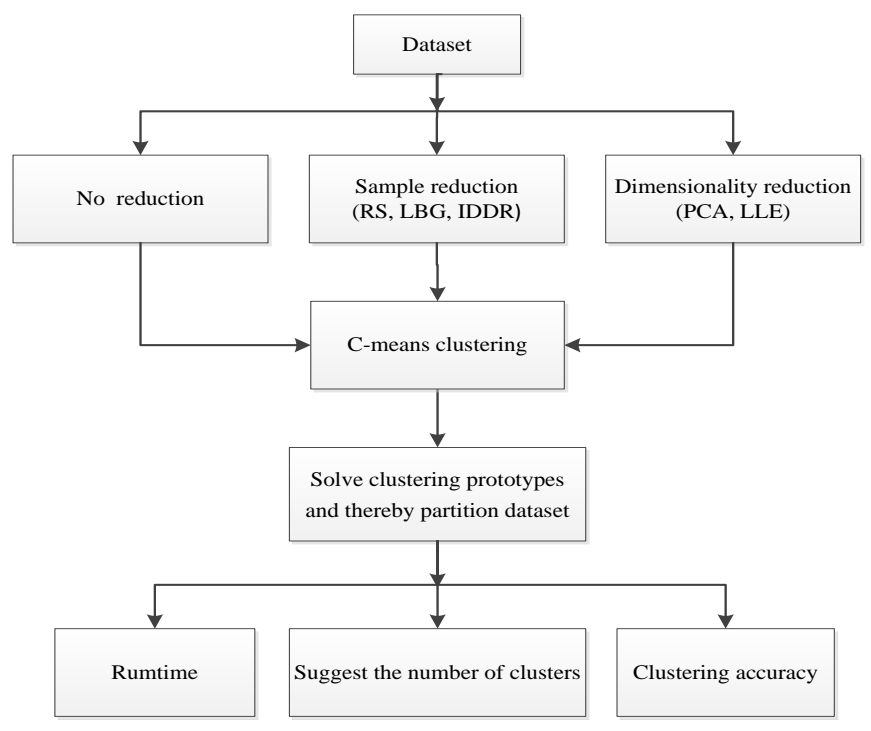

Fig.4. Experimental flowchart

\subsection{Test on three synthetic datasets}

The three synthetic datasets Set 1-Set 3 in Fig.5 are applied to visually show the reduction results of these three reduction methods. These three datasets have different densities, sizes and overlaps among various clusters, respectively. Tables 6-8 visually show the reduction results in Set 1-Set 3, respectively.

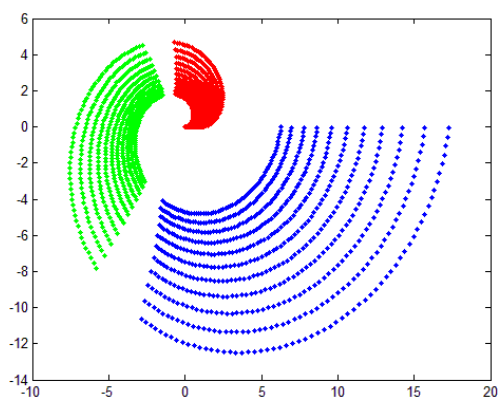

(a) Set 1

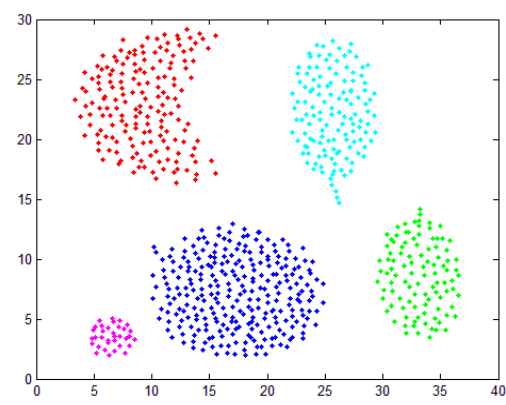

(b) Set 2



(c) Set 3

Fig.5. Three synthetic datasets

Table 6 Reduction results in Set 1 by RS, LBG, and IDDR algorithms.

\begin{tabular}{|c|c|c|c|}
\hline Algorithm & $50 \%$ reduction & $\mathbf{7 0 \%}$ reduction & $90 \%$ reduction \\
\hline \multicolumn{4}{|l|}{$\mathrm{RS}$} \\
\hline \multicolumn{4}{|l|}{ LBG } \\
\hline IDDR & & & \\
\hline & 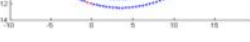 & 证 & at \\
\hline
\end{tabular}


Table 7 Reduction results in Set 2 by RS, LBG, and IDDR algorithms.

\begin{tabular}{|c|c|c|c|}
\hline Algorithm & $\mathbf{5 0 \%}$ reduction & $70 \%$ reduction & $90 \%$ reduction \\
\hline \multicolumn{4}{|l|}{$\mathrm{RS}$} \\
\hline \multicolumn{4}{|l|}{ LBG } \\
\hline IDDR & & & \\
\hline & . & 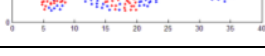 & in \\
\hline
\end{tabular}

Table 8 Reduction results in Set 3 by RS, LBG, and IDDR algorithms.

\begin{tabular}{|c|c|c|c|}
\hline Algorithm & $50 \%$ reduction & $70 \%$ reduction & $90 \%$ reduction \\
\hline \multicolumn{4}{|l|}{ RS } \\
\hline LBG & & & $7 x$ \\
\hline IDDR & & & H \\
\hline & 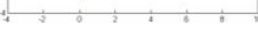 & 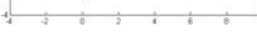 & 4 \\
\hline
\end{tabular}

In Tables 6-8, the original data points are blue and the remained data points are red. As the reduction rate increases, the remained data gradually tend to the cluster centers, and the boundary between clusters becomes clearer and clearer. Consequently, the cluster separations are enhanced and any clustering algorithm can easily find all the clusters. These results are desirable in any data reduction process, while RS and LBG cannot achieve these results well.

\subsection{4 real UCI datasets}

We choose 24 real datasets of different characteristics from UCI to test the accuracy and runtime of IDDR comparing to RS and LBG. According to the most dominant characteristic of any dataset, these tested datasets are partitioned into three classes: cluster size-different, cluster density-different and clusteroverlapped datasets. Table 9 shows the main characteristics of the three classes of datasets.

1) Cluster size-different dataset: The representative of the class of dataset is Ecoli, the maximal cluster consists of 143 data, and minimal cluster only contains 2 data. The cluster sizes of all eight clusters in Ecoli are $0.95,0.85,1.36,1.26,0.69,0.57,0.36$, and 0.74 . Therefore, it is difficult to keep the completeness of all clusters in the data reduction process. And the number of clusters is regarded as 5 in the experiment of 10 
validity index. Similar to Ecoli, the other seven datasets are User, Seeds, Hayes, Vehicle, Statlog, Parkinsons, and Segmentation.

2) Cluster density-different dataset: The representative of the class of dataset is Wpbc, which contains only two clusters, but the densities of the two clusters are very different. Such a dataset cannot be efficiently reduced by a unifying threshold. Similar to Wpbc, the other seven datasets are Wholesale, Tea, Spectf, Glass, Winequality, Transfusion, and Yeast.

3) Cluster-Overlapped dataset: The representative of the class of dataset is Iris. This three-cluster dataset IRIS contains 150 data with 4 attributes. Each cluster has 50 data. Two clusters are overlapped and one cluster is linearly separable from those two clusters. In past decades, the dataset has been frequently applied to evaluate the clustering results and the correctness of the number of clusters [33, 34]. Other seven IRIS-like datasets are Pima, Letter, Cancer, Movement, Vowel, BalanceS, and Pendigits.

Table 924 real datasets of UCI

\begin{tabular}{|c|c|c|c|c|c|c|c|c|c|c|c|}
\hline \multicolumn{4}{|c|}{ Cluster size-different dataset } & \multicolumn{3}{c|}{ Cluster density-different dataset } & \multicolumn{4}{c|}{ Overlapped cluster dataset } \\
\hline name & Data & Attribute & C & Name & Data & Attribute & C & Name & Data & Attribute & C \\
\hline User & 258 & 5 & 4 & Wholesale & 440 & 7 & 2 & Pima & 768 & 8 & 2 \\
\hline Seeds & 210 & 7 & 3 & Wpbc & 194 & 33 & 2 & Iris & 150 & 4 & 3 \\
\hline Hayes & 132 & 5 & 3 & Tea & 151 & 5 & 3 & Letter & 2000 & 16 & 26 \\
\hline Vehicle & 846 & 18 & 4 & Spectf & 267 & 44 & 2 & Cancer & 683 & 9 & 2 \\
\hline Ecoli & 336 & 7 & 5 & Glass & 214 & 9 & 6 & Movement & 360 & 90 & 15 \\
\hline Statlog & 270 & 13 & 2 & Winequality & 1599 & 11 & 4 & Vowel & 990 & 10 & 11 \\
\hline Parkinsons & 195 & 22 & 2 & Transfusion & 748 & 4 & 2 & BalanceS & 625 & 4 & 3 \\
\hline Segmentation & 2310 & 19 & 7 & Yeast & 1484 & 8 & 9 & Pendigits & 3498 & 16 & 10 \\
\hline
\end{tabular}

\subsection{Evaluation of the reduction results on 24 real datasets}

The data reduction results using the three methods on the real 24 datasets are characterized according to their clustering prototypes, separation, and runtime. 'Original' or '0\%' refer to the original datasets; '50\%', '70\%', '90\%' refer to the datasets being reduced by 50\%, 70\%, 90\%, respectively.

1) Accuracy of clustering prototype. A clustering prototype is the point with highest density in any cluster. In the $C$-MEANS algorithm, the clustering prototypes can perfectly determine clustering results. However, the location of the clustering prototype in the original dataset can be affected by sizes, densities, and separations among clusters, as well as the noisy data. These aspects will then help deduce the clustering accuracy. To determine accurately the clustering prototypes, we first reduce $50 \%, 70 \%$, $90 \%$ of the total amount in any dataset by turns, then determine the clustering prototypes in the reduced datasets, and finally assign all original data by these new clustering prototypes. The clustering results are in Table 10. IDDR has higher accuracy in almost all datasets at these reducing rates, while RS and LBG can barely increase their accuracies. Essentially, the increments of accuracy in LBG and RS apparently are less than those in IDDR. So IDDR can efficiently reduce the irrelevant and interfering data and determine more accurate clustering prototypes. However, IDDR is not always the best one. In these 4 ineffectual datasets of IDDR, Spectf and Balance $S$ showed the shapes of clusters in original datasets are arbitrary and there are no clear boundaries among different clusters. Consequently, the clustering results of these datasets by $C$-MEANS are not good since C-MEANS is originally designed to just find only spherical clusters. So these datasets are difficult to justify any method. Additionally, some datasets contain trivial clusters. For example, the dataset Ecoli has 8 clusters in which three clusters contains only 5, 3, and 2 data, respectively. Thus these clusters are lightly reduced and the corresponding cluster accuracy is not good enough.

2) Separation among clusters. If all clusters in a dataset are separated well, all data in the dataset can correctly be clustered. Otherwise, the clustering accuracy will decrease greatly. Thus the clustering accuracy 
after data reduction can represent the separation among the clusters. The columns of $50 \%, 70 \%$, and $90 \%$ in Table 10 shows the clustering accuracy after data reduction at the reduction rates of $50 \%, 70 \%$, and $90 \%$ using the three methods. The "total error" row at the bottom of Table 10 is the total number of datasets with decreased accuracy after data reduction. IDDR dominantly takes advantages over RS and LBG. Consequently, IDDR can effectively increase the separations among clusters in most datasets by removing the overlapped parts among the clusters. Essentially, the accuracy increments are very large in the four datasets Wholesale, Glass, Letter and Movement after using IDDR. As a comparison, RS can increase separation in only few datasets. LBG is a bit more accurate than RS but still large errors remain. Thus IDDR is very useful for increasing the separation of clusters. The effect is very helpful to many data mining fields.

Table 10 Clustering accuracy of 24 real datasets by C-MEANS

\begin{tabular}{|c|c|c|c|c|c|c|c|c|c|c|}
\hline \multicolumn{2}{|c|}{ Algorithm } & \multicolumn{3}{|c|}{$\mathbf{R S}$} & \multicolumn{3}{|c|}{ LBG } & \multicolumn{3}{|c|}{ IDDR } \\
\hline Name & Original & $50 \%$ & $70 \%$ & $90 \%$ & $50 \%$ & $70 \%$ & $90 \%$ & $50 \%$ & $70 \%$ & $90 \%$ \\
\hline User & 0.457 & -0.062 & +0.013 & -0.111 & -0.029 & +0.118 & +0.011 & +0.124 & +0.049 & +0.263 \\
\hline Seeds & 0.890 & +0.015 & +0.016 & -0.271 & +0.017 & +0.038 & -0.265 & +0.053 & +0.094 & +0.062 \\
\hline Hayes & 0.416 & -0.038 & +0.220 & -0.059 & -0.046 & +0.054 & -0.229 & +0.039 & +0.020 & +0.353 \\
\hline Vehicle & 0.365 & -0.230 & -0.040 & -0.083 & -0.140 & -0.149 & -0.029 & +0.051 & +0.022 & +0.052 \\
\hline Ecoli & 0.595 & -0.018 & -0.061 & -0.154 & -0.183 & -0.137 & -0.158 & -0.053 & -0.065 & +0.284 \\
\hline Statlog & 0.796 & -0.003 & -0.400 & -0.463 & +0.001 & +0.022 & +0.104 & +0.026 & -0.006 & -0.240 \\
\hline Parkinsons & 0.631 & -0.152 & -0.182 & -0.031 & -0.235 & -0.063 & -0.006 & +0.008 & +0.041 & +0.264 \\
\hline Segmentation & 0.582 & -0.466 & -0.445 & -0.448 & -0.458 & -0.473 & -0.444 & +0.175 & +0.092 & +0.240 \\
\hline Wholesale & 0.564 & +0.009 & -0.028 & +0.026 & -0.013 & -0.024 & -0.116 & +0.236 & +0.284 & +0.345 \\
\hline$W p b c$ & 0.592 & -0.118 & +0.061 & +0.058 & -0.077 & +0.044 & -0.059 & +0.027 & +0.063 & +0.092 \\
\hline $\mathrm{Tea}$ & 0.424 & -0.003 & -0.056 & +0.138 & -0.021 & -0.040 & +0.263 & +0.085 & +0.157 & +0.376 \\
\hline Spectf & 0.625 & -0.021 & -0.088 & +0.115 & +0.049 & +0.067 & -0.108 & -0.076 & +0.050 & +0.106 \\
\hline Glass & 0.435 & +0.022 & -0.047 & -0.254 & +0.065 & +0.110 & +0.103 & +0.282 & +0.438 & +0.279 \\
\hline Winequality & 0.271 & -0.004 & -0.011 & +0.060 & +0.031 & +0.064 & +0.147 & +0.052 & +0.157 & +0.366 \\
\hline Transfusion & 0.588 & +0.021 & +0.032 & +0.025 & -0.072 & -0.082 & -0.049 & +0.105 & +0.079 & +0.072 \\
\hline Yeast & 0.304 & +0.053 & +0.124 & +0.139 & +0.053 & +0.098 & +0.175 & +0.087 & +0.059 & +0.020 \\
\hline Pima & 0.668 & -0.171 & -0.116 & -0.032 & +0.016 & -0.020 & -0.059 & +0.090 & +0.136 & +0.200 \\
\hline Iris & 0.886 & -0.006 & -0.308 & -0.353 & -0.043 & -0.042 & -0.136 & -0.064 & -0.091 & +0.114 \\
\hline Letter & 0.170 & -0.040 & -0.042 & -0.070 & -0.085 & -0.070 & -0.078 & +0.244 & +0.323 & +0.476 \\
\hline Cancer & 0.960 & +0.004 & -0.007 & +0.011 & -0.038 & -0.011 & -0.069 & +0.009 & +0.040 & +0.040 \\
\hline Movement & 0.281 & -0.015 & -0.048 & -0.198 & -0.164 & -0.127 & -0.094 & +0.325 & +0.406 & +0.234 \\
\hline Vowel & 0.271 & +0.032 & -0.021 & -0.059 & -0.045 & -0.076 & -0.129 & +0.117 & +0.217 & +0.244 \\
\hline BalanceS & 0.493 & -0.017 & +0.054 & +0.031 & +0.148 & +0.188 & +0.132 & -0.070 & +0.109 & +0.088 \\
\hline Pendigits & 0.682 & -0.022 & -0.171 & -0.102 & +0.074 & -0.012 & -0.001 & +0.119 & +0.066 & +0.031 \\
\hline Total error & & 17 & 17 & 15 & 15 & 14 & 17 & 4 & 3 & 1 \\
\hline
\end{tabular}

Note: To highlight the contrast results, the sign ' + ' and '-' are used to represent the positive and negative increment of accuracy compared with the original datasets, respectively. The greater the positive increment, the greater the correct rate.

3) Running time. Table 11 shows the averaging time of data reduction using the three methods for 24 tested datasets.

Table 11 Runtime of 24 real datasets by RS, LBG and IDDR

\begin{tabular}{|l|c|c|c|c|c|c|c|c|c|}
\hline Algorithm & \multicolumn{4}{|c|}{ RS } & \multicolumn{3}{|c|}{ LBG } & \multicolumn{3}{|c|}{ IDDR } \\
\hline \multicolumn{1}{|c|}{ Name } & $\mathbf{5 0 \%}$ & $\mathbf{7 0 \%}$ & $\mathbf{9 0 \%}$ & $\mathbf{5 0 \%}$ & $\mathbf{7 0 \%}$ & $\mathbf{9 0 \%}$ & $\mathbf{5 0 \%}$ & $\mathbf{7 0 \%}$ & $\mathbf{9 0 \%}$ \\
\hline User & 0.001 & 0.000 & 0.000 & 0.221 & 0.120 & 0.054 & 0.002 & 0.002 & 0.002 \\
\hline Seeds & 0.001 & 0.000 & 0.000 & 0.154 & 0.146 & 0.078 & 0.002 & 0.001 & 0.001 \\
\hline Hayes & 0.000 & 0.000 & 0.000 & 0.074 & 0.041 & 0.023 & 0.001 & 0.001 & 0.001 \\
\hline Vehicle & 0.012 & 0.004 & 0.001 & 4.032 & 2.872 & 1.067 & 0.006 & 0.006 & 0.006 \\
\hline Ecoli & 0.006 & 0.001 & 0.000 & 0.425 & 0.208 & 0.158 & 0.002 & 0.002 & 0.002 \\
\hline Statlog & 0.001 & 0.001 & 0.000 & 0.629 & 0.390 & 0.074 & 0.002 & 0.002 & 0.002 \\
\hline Parkinsons & 0.001 & 0.005 & 0.000 & 0.287 & 0.191 & 0.177 & 0.001 & 0.001 & 0.001 \\
\hline Segmentation & 0.003 & 0.002 & 0.000 & 0.736 & 0.713 & 0.215 & 0.004 & 0.003 & 0.003 \\
\hline Wholesale & 0.009 & 0.001 & 0.000 & 0.687 & 0.773 & 0.307 & 0.003 & 0.003 & 0.003 \\
\hline Wpbc & 0.001 & 0.001 & 0.000 & 0.470 & 0.299 & 0.080 & 0.001 & 0.001 & 0.001 \\
\hline Tea & 0.000 & 0.000 & 0.000 & 0.035 & 0.021 & 0.019 & 0.001 & 0.001 & 0.001 \\
\hline Spectf & 0.003 & 0.001 & 0.000 & 7.174 & 0.800 & 0.122 & 0.002 & 0.002 & 0.002 \\
\hline Glass & 0.001 & 0.000 & 0.000 & 0.165 & 0.055 & 0.035 & 0.001 & 0.001 & 0.001 \\
\hline Winequality & 0.020 & 0.006 & 0.001 & 7.693 & 3.770 & 2.639 & 0.010 & 0.009 & 0.009 \\
\hline Transfusion & 0.005 & 0.001 & 0.000 & 0.641 & 0.625 & 0.314 & 0.003 & 0.003 & 0.004 \\
\hline Yeast & 0.013 & 0.005 & 0.001 & 8.556 & 5.570 & 4.195 & 0.010 & 0.010 & 0.010 \\
\hline Pima & 0.004 & 0.002 & 0.000 & 1.860 & 1.009 & 0.890 & 0.005 & 0.005 & 0.005 \\
\hline Iris & 0.000 & 0.000 & 0.000 & 0.080 & 0.076 & 0.018 & 0.001 & 0.001 & 0.001 \\
\hline Letter & 0.013 & 0.004 & 0.001 & 5.197 & 5.008 & 0.926 & 0.007 & 0.007 & 0.007 \\
\hline Cancer & 0.002 & 0.001 & 0.000 & 0.813 & 0.654 & 0.142 & 0.006 & 0.006 & 0.004 \\
\hline Movement & 0.005 & 0.003 & 0.000 & 2.361 & 0.829 & 0.603 & 0.003 & 0.003 & 0.002 \\
\hline Vowel & 0.006 & 0.007 & 0.001 & 2.070 & 2.205 & 0.618 & 0.007 & 0.007 & 0.007 \\
\hline BalanceS & 0.001 & 0.000 & 0.000 & 0.372 & 0.211 & 0.062 & 0.002 & 0.002 & 0.002 \\
\hline Pendigits & 0.007 & 0.009 & 0.001 & 2.486 & 2.480 & 1.088 & 0.006 & 0.006 & 0.006 \\
\hline
\end{tabular}

RS only performs a series of inquiry operations and thus its runtime of the data reduction process tends 
towards zero when compared with LBG and IDDR. The runtime of LBG is much higher than IDDR since the time complexity is a quantity grade higher than LBG. But the clustering time is least after performing IDDR among the three reduction algorithms since most datasets have better separations after data reduction. The clustering time of LBG for the reducing datasets with respect to RS is longest since the structural information of these datasets is hardly found. So IDDR is very valuable for data reduction.

4) Clustering validity. Table 12 shows the accuracy of the five typical validity indexes $\mathrm{CH}, \mathrm{DB}, \mathrm{PB}$, Sil, and KL on the 24 datasets, the bottom row shows the total number of correct suggestions by these indexes. Thereby we can deduce the following results:

- Compared with the original datasets, the accuracy of these validity indexes totally are higher, essentially the PB index. This demonstrates that the reduced dataset has the better separation among clusters. But in the partial dataset, the reduced dataset is as same bad as the original dataset or worse.

- Different validity indexes have different advantages over the other validity indexes. We think there are three reasons to explain this. Firstly, C-MEANS clustering have randomness, and different initializations have very different clustering results. Secondly, each validity index has limited applicable range and greatly is affected by different data structures. Finally, the C-MEANS cannot correctly identify non-spherical clusters. Consequently, no validity can efficiently evaluate all clustering results.

Table 12 Results of clustering validity indexes on 24 real datasets

\begin{tabular}{|c|c|c|c|c|c|c|c|c|c|c|c|c|c|c|c|c|c|c|c|c|}
\hline \multirow{2}{*}{$\begin{array}{c}\text { Algorithm } \\
\text { Name }\end{array}$} & \multicolumn{4}{|c|}{ CH } & \multicolumn{4}{|c|}{ DB } & \multicolumn{4}{|c|}{ PB } & \multicolumn{4}{|c|}{ Sil } & \multicolumn{4}{|c|}{ KL } \\
\hline & $0 \%$ & $\mathbf{5 0 \%}$ & $70 \%$ & $90 \%$ & $0 \%$ & $\mathbf{5 0 \%}$ & $70 \%$ & $\mathbf{9 0 \%}$ & $0 \%$ & $\mathbf{5 0 \%}$ & $70 \%$ & $\mathbf{9 0 \%}$ & $0 \%$ & $\mathbf{5 0 \%}$ & $70 \%$ & $\mathbf{9 0 \%}$ & $0 \%$ & $50 \%$ & $70 \%$ & $90 \%$ \\
\hline User & $2 / 4$ & $2 / 4$ & $3 / 4$ & $3 / 4$ & $6 / 4$ & $3 / 4$ & $9 / 4$ & $9 / 4$ & $2 / 4$ & $2 / 4$ & $2 / 4$ & $3 / 4$ & $2 / 4$ & $2 / 4$ & $3 / 4$ & $3 / 4$ & $10 / 4$ & $7 / 4$ & $2 / 4$ & $2 / 4$ \\
\hline Seeds & $2 / 3$ & $3 / 3$ & $3 / 3$ & $9 / 3$ & $2 / 3$ & $3 / 3$ & $3 / 3$ & $10 / 3$ & $2 / 3$ & $2 / 3$ & $2 / 3$ & $2 / 3$ & $2 / 3$ & $2 / 3$ & $3 / 3$ & $9 / 3$ & $2 / 3$ & $2 / 3$ & $2 / 3$ & $2 / 3$ \\
\hline Hayes & $2 / 3$ & $2 / 3$ & $2 / 3$ & $10 / 3$ & $10 / 3$ & $10 / 3$ & $9 / 3$ & $9 / 3$ & $2 / 3$ & $2 / 3$ & $2 / 3$ & $2 / 3$ & $2 / 3$ & $2 / 3$ & $7 / 3$ & $10 / 3$ & $4 / 3$ & $3 / 3$ & $3 / 3$ & $2 / 3$ \\
\hline Vehicle & $2 / 4$ & $2 / 4$ & $2 / 4$ & $3 / 4$ & $2 / 4$ & $2 / 4$ & $2 / 4$ & $8 / 4$ & $2 / 4$ & $2 / 4$ & $2 / 4$ & $2 / 4$ & $2 / 4$ & $2 / 4$ & $2 / 4$ & $3 / 4$ & $2 / 4$ & $2 / 4$ & $2 / 4$ & $2 / 4$ \\
\hline Ecoli & $3 / 5$ & $4 / 5$ & $3 / 5$ & $6 / 5$ & $6 / 5$ & $4 / 5$ & $8 / 5$ & $9 / 5$ & $2 / 5$ & $2 / 5$ & $2 / 5$ & $2 / 5$ & $3 / 5$ & $2 / 5$ & $6 / 5$ & $9 / 5$ & $2 / 5$ & $3 / 5$ & $2 / 5$ & $2 / 5$ \\
\hline Statlog & $2 / 2$ & $2 / 2$ & $6 / 2$ & $3 / 2$ & $7 / 2$ & $5 / 2$ & $5 / 2$ & $10 / 2$ & $2 / 2$ & $2 / 2$ & $2 / 2$ & $2 / 2$ & $8 / 2$ & $6 / 2$ & $6 / 2$ & $3 / 2$ & $2 / 2$ & $2 / 2$ & $2 / 2$ & $2 / 2$ \\
\hline Parkinsons & $2 / 2$ & $2 / 2$ & $3 / 2$ & $2 / 2$ & $9 / 2$ & $7 / 2$ & $10 / 2$ & $10 / 2$ & $2 / 2$ & $2 / 2$ & $2 / 2$ & $2 / 2$ & $2 / 2$ & $2 / 2$ & $3 / 2$ & $6 / 2$ & $2 / 2$ & $2 / 2$ & $2 / 2$ & $2 / 2$ \\
\hline Segmentation & $2 / 7$ & $6 / 7$ & $7 \pi$ & $10 / 7$ & $4 / 7$ & $5 / 7$ & $4 / 7$ & $9 / 7$ & $2 / 7$ & $2 / 7$ & $2 / 7$ & $2 / 7$ & $2 / 7$ & $6 / 7$ & $7 / 7$ & $9 / 7$ & $2 / 7$ & $2 / 7$ & $2 / 7$ & $2 / 7$ \\
\hline Wholesale & $3 / 2$ & $2 / 2$ & $2 / 2$ & $2 / 2$ & $6 / 2$ & $2 / 2$ & $2 / 2$ & $2 / 2$ & $8 / 2$ & $2 / 2$ & $3 / 2$ & $2 / 2$ & $3 / 2$ & $2 / 2$ & $2 / 2$ & $2 / 2$ & $3 / 2$ & $2 / 2$ & $3 / 2$ & $3 / 2$ \\
\hline$W p b c$ & $2 / 2$ & $2 / 2$ & $2 / 2$ & $2 / 2$ & $8 / 2$ & $7 / 2$ & $10 / 2$ & $10 / 2$ & $4 / 2$ & $5 / 2$ & $2 / 2$ & $2 / 2$ & $2 / 2$ & $5 / 2$ & $2 / 2$ & $2 / 2$ & $9 / 2$ & $2 / 2$ & $2 / 2$ & $2 / 2$ \\
\hline Tea & $9 / 3$ & $7 / 3$ & $8 / 3$ & $7 / 3$ & $9 / 3$ & $7 / 3$ & $8 / 3$ & $7 / 3$ & $2 / 3$ & $2 / 3$ & $2 / 3$ & $2 / 3$ & $2 / 3$ & $5 / 3$ & $7 / 3$ & $7 / 3$ & $2 / 3$ & $4 / 3$ & $2 / 3$ & $8 / 3$ \\
\hline Spectf & $2 / 2$ & $2 / 2$ & $2 / 2$ & $2 / 2$ & $10 / 2$ & $9 / 2$ & $6 / 2$ & $8 / 2$ & $9 / 2$ & $2 / 2$ & $7 / 2$ & $8 / 2$ & $2 / 2$ & $2 / 2$ & $2 / 2$ & $2 / 2$ & $10 / 2$ & $9 / 2$ & $2 / 2$ & $2 / 2$ \\
\hline Glass & $2 / 6$ & $3 / 6$ & $3 / 6$ & $7 / 6$ & $2 / 6$ & $3 / 6$ & $8 / 6$ & $10 / 6$ & $2 / 6$ & $3 / 6$ & $2 / 6$ & $2 / 6$ & $2 / 6$ & $3 / 6$ & $3 / 6$ & $6 / 6$ & $3 / 6$ & $2 / 6$ & $2 / 6$ & $3 / 6$ \\
\hline Winequality & $2 / 4$ & $2 / 4$ & $2 / 4$ & $3 / 4$ & $2 / 4$ & $2 / 4$ & $5 / 4$ & $3 / 4$ & $2 / 4$ & $2 / 4$ & $3 / 4$ & $2 / 4$ & $2 / 4$ & $2 / 4$ & $2 / 4$ & $2 / 4$ & $6 / 4$ & $3 / 4$ & $8 / 4$ & $5 / 4$ \\
\hline Transfusion & $2 / 2$ & $2 / 2$ & $7 / 2$ & $5 / 2$ & $10 / 2$ & $4 / 2$ & $9 / 2$ & $9 / 2$ & $3 / 2$ & $2 / 2$ & $2 / 2$ & $2 / 2$ & $2 / 2$ & $4 / 2$ & $9 / 2$ & $5 / 2$ & $4 / 2$ & $2 / 2$ & $2 / 2$ & $2 / 2$ \\
\hline Yeast & $7 / 9$ & $10 / 9$ & $8 / 9$ & $6 / 9$ & $7 / 9$ & $9 / 9$ & $8 / 9$ & $8 / 9$ & $6 / 9$ & $10 / 9$ & $11 / 9$ & $8 / 9$ & $7 / 9$ & $8 / 9$ & $8 / 9$ & $8 / 9$ & $13 / 9$ & $6 / 9$ & $8 / 9$ & $10 / 9$ \\
\hline Pima & $2 / 2$ & $2 / 2$ & $2 / 2$ & $2 / 2$ & $5 / 2$ & $3 / 2$ & $2 / 2$ & $4 / 2$ & $2 / 2$ & $2 / 2$ & $2 / 2$ & $2 / 2$ & $2 / 2$ & $2 / 2$ & $2 / 2$ & $2 / 2$ & $10 / 2$ & $4 / 2$ & $8 / 2$ & $2 / 2$ \\
\hline Iris & $3 / 3$ & $2 / 3$ & $3 / 3$ & $7 / 3$ & $4 / 3$ & $2 / 3$ & $2 / 3$ & $8 / 3$ & $2 / 3$ & $2 / 3$ & $9 / 3$ & $5 / 3$ & $2 / 3$ & $2 / 3$ & $2 / 3$ & $2 / 3$ & $2 / 3$ & $2 / 3$ & $2 / 3$ & $2 / 3$ \\
\hline Letter & $22 / 26$ & $24 / 26$ & $24 / 26$ & $22 / 26$ & $29 / 26$ & $30 / 26$ & $26 / 26$ & $27 / 26$ & $30 / 26$ & $28 / 26$ & $27 / 26$ & $26 / 26$ & $27 / 26$ & $25 / 26$ & $22 / 26$ & $28 / 26$ & $30 / 26$ & $30 / 26$ & $27 / 26$ & $27 / 26$ \\
\hline Cancer & $2 / 2$ & $2 / 2$ & $2 / 2$ & $2 / 2$ & $2 / 2$ & $2 / 2$ & $2 / 2$ & $2 / 2$ & $7 / 2$ & $2 / 2$ & $2 / 2$ & $2 / 2$ & $2 / 2$ & $2 / 2$ & $2 / 2$ & $2 / 2$ & $10 / 2$ & $6 / 2$ & $6 / 2$ & $2 / 2$ \\
\hline Movement & $12 / 15$ & $11 / 15$ & $13 / 15$ & $17 / 15$ & $19 / 15$ & $14 / 15$ & $13 / 15$ & $19 / 15$ & $19 / 15$ & $17 / 15$ & $14 / 15$ & $14 / 15$ & $19 / 15$ & $13 / 15$ & $13 / 15$ & $17 / 15$ & $17 / 15$ & $11 / 15$ & $14 / 15$ & $12 / 15$ \\
\hline Vowel & $7 / 11$ & $12 / 11$ & $8 / 11$ & $7 / 11$ & $15 / 11$ & $13 / 11$ & $14 / 11$ & $15 / 11$ & $15 / 11$ & $14 / 11$ & $15 / 11$ & $10 / 11$ & $7 / 11$ & $12 / 11$ & $9 / 11$ & $11 / 11$ & $15 / 11$ & $14 / 11$ & $10 / 11$ & $11 / 11$ \\
\hline BalanceS & $4 / 3$ & $4 / 3$ & $3 / 3$ & $3 / 3$ & $8 / 3$ & $5 / 3$ & $7 / 3$ & $6 / 3$ & $2 / 3$ & $2 / 3$ & $2 / 3$ & $2 / 3$ & $4 / 3$ & $4 / 3$ & $3 / 3$ & $3 / 3$ & $3 / 3$ & $2 / 3$ & $2 / 3$ & $2 / 3$ \\
\hline Pendigits & $7 / 10$ & $6 / 10$ & $7 / 10$ & $8 / 10$ & $8 / 10$ & $9 / 10$ & $9 / 10$ & $9 / 10$ & $6 / 10$ & $14 / 10$ & $7 / 10$ & $6 / 10$ & $7 / 10$ & $6 / 10$ & $7 / 10$ & $9 / 10$ & $6 / 10$ & $7 / 10$ & $6 / 10$ & $6 / 10$ \\
\hline Correct NC & 17 & 21 & 21 & 17 & 9 & 23 & 20 & 15 & 16 & 23 & 23 & 23 & 16 & 19 & 19 & 17 & 14 & 21 & 21 & 21 \\
\hline
\end{tabular}

Note: results are given by the form ' $x / y$ ' with marks, therein, $x$ is the suggested number of cluster by validity indexes and $y$ is the actual number of cluster. Results that $x$ is equal to or close to $y$ are marked by the gray shading.

\subsection{Comparison of the performance on data reduction and dimensionality reduction}

Dimensionality reduction and sample reduction both are used to reduce a dataset to find the key structures. To test the performance of our method, we used it and other two typical dimensionality reduction methods PCA and LLE to analyze 24 datasets. In order to observe entire reducing performance, the reduction rates in PCA and LLE are taken as 10\%, 30\%, 50\%, 70\% and 90\%, respectively. The clustering accuracy of C-MEANS in the reduced datasets is used to test whether these reduction algorithms can capture the main data structures hidden in a large amount of data. Experimental results are shown in Table 13 below.

Table 13 shows PCA possesses better performances on clustering density-different datasets than on the clustering the other two classes of datasets. In addition, PCA can work better in low reducing rates than in higher ones. This indicates that the neglected component may contain important information. More importantly, no low-dimensional dataset can be clustered much better by PCA dimensionality reduction. On the other hand, although the overall accuracies of LLE are not good enough, its performances on the density-size datasets are relatively better. Data in low-dimensional space can preserve the topological structure and are easy to be clustered or classified. The worse results may be caused by the inappropriate numbers of neighbors that are difficult to be determined. 
In summary, IDDR has higher accuracy in almost all tested datasets compared with Table 10, while PCA and LLE only increase their accuracies just in minority. The results don't mean that sample reduction is more efficient than dimensionality reduction. In fact, the used features (dimension) in most of these 24 real tested datasets have been pre-selected, so that they are difficulty to be further reduced. However, in these datasets there are still irrelevant, inconsistent and redundant data that not only cost extra storage and memory space, but also compromise classification accuracy and decision correctness. In the opinions of cluster analysis, these irrelevant and redundant data are characterized as density-different, size-different and overlapped clusters. Therefore, sample reduction is necessary and supposed to be more efficient. Sample reduction plays its roles in reducing these datasets including irrelevant, inconsistent and redundant data while the dimensionality reduction is used for feature extraction, and contradictory information, and etc. They have different applicable ranges

Table 13 Clustering accuracy of 24 real datasets by C-MEANS

\begin{tabular}{|c|c|c|c|c|c|c|c|c|c|c|}
\hline Algorithm & \multicolumn{5}{|c|}{ PCA } & \multicolumn{5}{|c|}{ LLE } \\
\hline Name & $10 \%$ & $30 \%$ & $50 \%$ & 70\% & $90 \%$ & $10 \%$ & $30 \%$ & $\mathbf{5 0 \%}$ & $70 \%$ & $90 \%$ \\
\hline User & -0.050 & -0.039 & +0.000 & +0.000 & -0.050 & +0.081 & -0.039 & -0.101 & -0.105 & -0.097 \\
\hline Seeds & +0.005 & -0.062 & -0.062 & -0.062 & -0.062 & -0.081 & -0.052 & -0.052 & -0.052 & -0.052 \\
\hline Hayes & -0.030 & -0.091 & -0.068 & -0.045 & -0.030 & +0.045 & -0.061 & +0.015 & +0.000 & +0.015 \\
\hline Vehicle & +0.006 & +0.001 & +0.048 & +0.048 & +0.051 & +0.014 & +0.041 & -0.100 & -0.100 & -0.100 \\
\hline Ecoli & -0.018 & -0.018 & -0.042 & -0.161 & -0.155 & +0.018 & -0.015 & -0.128 & -0.226 & -0.226 \\
\hline Statlog & +0.000 & +0.000 & +0.000 & -0.004 & -0.019 & -0.256 & -0.267 & -0.167 & -0.144 & -0.178 \\
\hline Parkinsons & +0.000 & +0.000 & -0.036 & -0.036 & -0.036 & -0.015 & -0.108 & -0.144 & -0.144 & -0.144 \\
\hline Segmentation & +0.043 & +0.036 & -0.060 & -0.061 & -0.048 & -0.124 & -0.248 & -0.209 & -0.163 & -0.070 \\
\hline Wholesale & -0.009 & -0.007 & +0.007 & -0.005 & -0.005 & -0.077 & -0.077 & -0.077 & -0.077 & -0.077 \\
\hline$W p b c$ & -0.077 & -0.046 & +0.000 & -0.046 & +0.005 & +0.124 & +0.005 & -0.036 & -0.036 & -0.067 \\
\hline Tea & -0.040 & +0.000 & -0.119 & -0.033 & -0.033 & -0.099 & +0.013 & -0.053 & -0.099 & -0.099 \\
\hline Spectf & -0.004 & -0.004 & +0.000 & +0.000 & +0.000 & +0.071 & +0.034 & +0.041 & +0.030 & +0.030 \\
\hline Glass & +0.014 & +0.056 & +0.014 & +0.014 & +0.042 & -0.014 & -0.019 & -0.061 & -0.061 & -0.065 \\
\hline Winequality & -0.005 & +0.008 & +0.003 & -0.048 & -0.095 & -0.100 & -0.110 & -0.107 & -0.104 & -0.114 \\
\hline Transfusion & +0.007 & +0.007 & +0.009 & +0.011 & +0.011 & +0.000 & -0.005 & -0.011 & -0.011 & -0.011 \\
\hline Yeast & +0.006 & -0.059 & -0.087 & -0.082 & -0.128 & -0.129 & -0.145 & -0.214 & -0.218 & -0.228 \\
\hline Pima & +0.000 & -0.003 & +0.000 & -0.003 & +0.016 & -0.023 & -0.023 & -0.023 & -0.023 & -0.023 \\
\hline Iris & +0.000 & +0.093 & +0.093 & +0.093 & +0.093 & -0.160 & -0.167 & -0.167 & -0.167 & -0.167 \\
\hline Letter & -0.036 & -0.075 & -0.128 & -0.174 & -0.245 & -0.051 & -0.066 & -0.105 & -0.126 & -0.204 \\
\hline Cancer & +0.001 & +0.003 & +0.000 & +0.000 & +0.000 & -0.061 & -0.213 & -0.216 & -0.216 & -0.216 \\
\hline Movement & -0.006 & -0.058 & -0.078 & -0.156 & -0.189 & -0.017 & -0.011 & -0.039 & -0.133 & -0.172 \\
\hline Vowel & +0.016 & +0.056 & +0.003 & +0.018 & -0.091 & -0.081 & -0.076 & -0.081 & -0.090 & -0.078 \\
\hline BalanceS & -0.008 & +0.000 & -0.054 & -0.069 & -0.048 & -0.038 & +0.034 & -0.072 & -0.072 & -0.062 \\
\hline Pendigits & +0.000 & -0.039 & -0.107 & -0.317 & -0.320 & -0.102 & -0.168 & -0.312 & -0.282 & -0.282 \\
\hline Total error & 11 & 12 & 11 & 16 & 16 & 17 & 19 & 22 & 22 & 22 \\
\hline
\end{tabular}

Note: the meaning of the sign ' + ' and '-' are same as that in Table 10.

\section{Conclusion}

We proposed an unsupervised data reduction method (IDDR) with the following four advantages. Firstly, different from other data reduction methods, IDDR applies only grid-based partition without any distance computation among data, which makes it have much lower time complexity than other algorithms. Secondly, IDDR doesn't require any user-specified parameters to reduce the time cost, while many other existing algorithms' efficiencies depend on their given parameters. In this point of view, IDDR is unsupervised and robust. Thirdly, for various datasets, IDDR has better ability to locate the meaningful subsets of representative examples in each cluster and to make the clusters have clear boundaries. Moreover, IDDR can provide more information about clustering. Finally, IDDR outperforms the class of dimensionality reduction methods and shows its superiority when the dimensionality reduction is invalid in raising the clustering accuracy. Our experiments strongly confirmed these advantages. Because of these promising performances, we believe that IDDR is a quite reasonable and applicable method to reduce much larger datasets.

The main drawback of IDDR is the bisecting way compared with RS and LVQ. For example, the alternative bisecting way may not be optimal. Further studies are needed to optimally improve efficiency on the basis of retaining the advantages of IDDR. 
Acknowledgments This work was supported by the National Science Foundation of China under Grant No. $60772080,61573251$.

\section{References}

[1] B. S. Everitt, The Cambridge Dictionary of Statistics, Cambridge University Press, New York, 2006.

[2] G. Sanguinetti, Dimensionality reduction of clustered data sets, IEEE Trans. Patt. Anal. Mach. Intell., 30(2008) 535-540.

[3] J. Tenenbaum, V. Silva, J. Langford, A global geometric framework for nonlinear dimensionality reduction, Science, 290(2000) 2319-2323.

[4]J. A. Costa, A.O. Hero, Geodesic entropic graphs for dimension and entropy estimation in manifold learning, IEEE Trans. Signal Process, 52 (2004) 2210-2221.

[5] X. Li, Data reduction via adaptive sampling, Communications in Information and Systems, 2 (2002) 53-68.

[6] L. Polkowski, Rough Set Theory: An Introduction, Advances in Soft computing, 15(2013) 3-92

[7] J. Shlens, A Tutorial on Principal Component Analysis, Int. J. Remote Sens., 51(2014)354-364.

[8] T. S. Roweis, L. K. Saul, Nonlinear Dimensionality Reduction by Locally Linear Embedding, Science, 290 (2000):2323-2326.

[9] C. Hong, J. Yu, J. Li, et al, Multi-view hypergraph learning by patch alignment framework, Neurocomputing, 118 (2013) 79-86.

[10] J. Yu, R. Hong, M. Wang, et al, Image clustering based on sparse patch alignment framework, Pattern Recognition, 47 (2014) 3512-3519.

[11] Y. Li, X. Shi, L. Tong, et al. Manifold Regularized Multi-view Feature Selection for Web Image Annotation, Neurocomputing, 204 (2016) 135-141.

[12] X. Li, V. S. Jacob, Adaptive data reduction for large-scale transaction data, European Journal of Operational Research, 188 (2008) 910-924.

[13] P. Qin , W. Xu, J. Guo, A novel negative sampling based on TFIDF for learning word representation, Neurocomputing, 177(2015)257-265.

[14] S. Fabrizio, Machine learning in automated text categorization, ACM Computing Surveys, 34(2002)1-47.

[15] D. Nebel, B. Hammer, K. Frohberg, et al, Median variants of learning vector quantization for learning of dissimilarity data, Neurocomputing, 169(2015)295-305.

[16] C. Domingo, R. Gavalda, O. Watanbe, Adaptive sampling methods for scaling up knowledge discovery algorithms, Data Mining and Knowledge Discovery, 6(2002) 131-152.

[17]Y. Linde, A. Buzo, and R. Gray, An algorithm for vector quantization design, IEEE Trans. Communications, 28 (1980) 84-95.

[18]U. Fayyad, R. Uthurusamy, Evolving data mining into solutions for insights, Communications of the ACM, 45(2002) 28-31.

[19]S. Yue, M. Wei, J. Wang, A general grid-based approach to clustering, Patt. Recog. Lett., 29(2008)434-441.

[20]M. Ester, H. P. Kriegel, J. Sander, X. Xu, A density-based algorithm for discovering clusters in large spatial databases with noise. Proc. the 2nd Int. Conf. KDD., (1996)226-231

[21]J. Wang, C. Lin, Y. Yang, A K-Nearest-Neighbor Classifier with Heart Rate Variability Feature-based Transformation Algorithm for Driving Stress Recognition, Neurocomputing, 116(2013) 136-143.

[22]J. MacQueen, Some methods for classification and analysis of multivariate observations, in The 5th Berkeley Symposium on Mathematical and Probability, Berkeley, 1967.

[23] F. Nie, X. Wang, H. Huang, Clustering and projected clustering with adaptive neighbors, ACM Knowledge Discovery and Data Mining, 2014, pp.977-986.

[24]J. Tantrum, A. Murua, W. Stuetzle, Hierarchical model-based clustering of large datasets through fractionation and refractionation, Information Systems, 29(2004)315-326. 
[25]R. Xu, D. C. Wunsch, Survey of clustering algorithm, IEEE Trans. Neural Networks, 16(2005) 645-661.

[26]Y. Wei, W. Xia, M. Lin, A Flexible CNN Framework for Multi-label Image Classification, IEEE Trans. Pattern Analysis and Machine Intelligence, 38 (2016)1901-1907.

[27] D. L. Davies, D.W. Bouldin, A cluster separation measure, IEEE Trans. Patt. Anal. Mach. Intell., 1 (1979) 224-227.

[28] M. K. Pakhira, S. Bandyopadhyay, U. Maulik, Validity index for crisp and fuzzy clusters, Pattern Recognition, 37 (2004) 487-501.

[29]T. Calinski, J. Harabasz, A dendrite method for cluster analysis, Communications in Statistics, 3(1974)1-27.

[30]P. Rousseeuw, Silhouettes: a graphical aid to the interpretation and validation of cluster analysis, Journal of Computational and Applied Mathematics, 20 (1987)53-65.

[31] W. J. Krzanowski, Y. T. Lai, A Criterion for Determining the Number of Groups in a Data Set Using Sum-of-Squares Clustering, Biometrics, 44(1988) 23-34.

[32] M. Lichman, UCI Machine Learning Repository [http://archive.ics.uci.edu/ml], Irvine, CA: University of California, School of Information and Computer Science, 2013.

[33] O. Arbelaitz, I. Gurrutxaga, J. Muguerza, et al, An extensive comparative study of cluster validity indices, Pattern Recognition, 46(2013) 243-256.

[34]S. Yue, J. Wang, J. Wang, et al, A new validity index for evaluating the clustering results by partitional clustering algorithms, Soft Computing, 20(2016) 1127-1138. 